\section{Interaction between cyclosporin and fluoxetine}

Dr R C Horton and Mr R S Bonser (Queen Elizabeth Hospital, Birmingham B15 2TH) write: We report an interaction between cyclosporin and fluoxetine causing a rise in cyclosporin concentrations.

A 59 year old man underwent cardiac transplantation for end stage heart failure. Cyclosporin was given as an immunosuppressant, and he was maintained on $225 \mathrm{mg}$ twice daily with a stable trough whole blood concentration of $300 \mu \mathrm{g} / \mathrm{l}$. Progress in hospital was satisfactory, but 17 days postoperatively he developed an acute depressive illness and was given fluoxetine $20 \mathrm{mg}$ once daily. No other changes were made to his drug treatment (azathioprine, prednisolone, ranitidine, aspirin amphotericin, and co-trimoxazole). After 10 days his cyclosporin concentration had risen to $588 \mu \mathrm{g} / \mathrm{l}$. We reduced the dose of cyclosporin to $75 \mathrm{mg}$ twice daily and his blood concentration then remained at $250 \mu \mathrm{g} / \mathrm{l}$. The patient did not respond to fluoxetine, so the drug was stopped. After seven days his cyclosporin concentration had fallen to $95 \mu \mathrm{g} / \mathrm{l}$, necessitating a dose increase to $200 \mathrm{mg}$ twice daily. His blood cyclosporin concentration then remained at $300 \mu \mathrm{g} / \mathrm{l}$. Hepatic and renal function were normal throughout.

Several drugs are known to interfere with the metabolism of cyclosporin. Those that induce hepatic cytochrome $P-450$ enzymes may reduce blood cyclosporin concentrations, while inhibitors of hepatic metabolism may increase concentrations. ${ }^{1}$ In our patien fluoxetine administration led to increased cyclosporin concentrations. This is probably a consequence of fluoxetine's potent inhibition of the cytochrome $P-450$ enzyme P450IID6. ${ }^{2}$ A less likely possibility is that fluoxetine increased the systemic availability of cyclosporin by changed absorption. Since

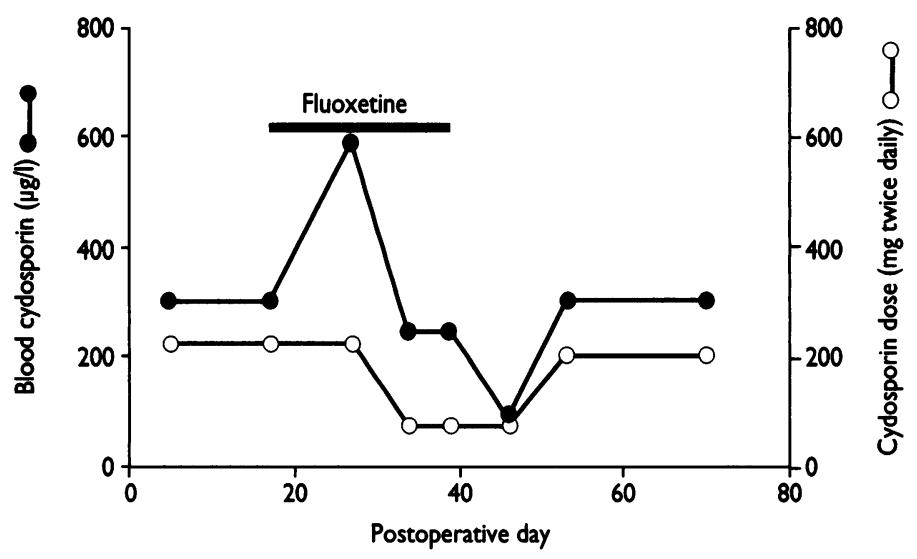

Cyclosporin dose and effect of fluoxetine on blood cyclosporin concentration

a whole blood (lysed cells) radioimmunoassay was used, altered binding of cyclosporin to red cells cannot be implicated.

Cyclosporin concentrations should be carefully monitored in patients who have undergone transplantation and are given fluoxetine. Raised concentrations may lead to cyclosporin related nephrotoxicity, ${ }^{3}$ while reduced concentrations may lead to inadequate immunosuppression and graft loss.

1 Scott JP, Higenbottam JW. Adverse reactions and interactions of cyclosporin. Med Toxicol 1988;3:107-27.

Brosen K, Skjelbo E. Fluoxetine and norfluoxetine are potent inhibitors of P340IID6-the source of the spateine/ debrisoquine oxidation polymorphism. $\mathrm{Br} \mathcal{F}$ Clin Pharmacol 1991;32:136-7.

Keown PA, Stiller CR, Laupacis AL, Howson W, Coles R, Stawecki M, et al. The effects and side effects of cyclosporin: relationship to drug pharmacokinetics. Transplant Proc $1982 ; 14: 659-61$

\section{Transverse myelitis after measles, mumps, and rubella vaccine}

Drs K A Joyce and J E ReEs (Hurstwood Park Neurological Centre, Haywards Heath, West Sussex RH16 4EX) write: A 20 year old man who planned to work with children in the United States was instructed to be vaccinated against rubella and was given measles, mumps, and rubella vaccine. Five days later he developed fever, malaise, sore throat, and a transient rash over his upper body. These symptoms fluctuated over the next two weeks, after which he developed urinary retention, followed by an ascending paraesthesia. On admission to hospital he had a rapidly progressive flaccid paraplegia, with sensory loss below $\mathrm{T} 1$ and loss of anal tone. He was feverish and remained so for 36 hours.

Full blood count and erythrocyte sedimentation rate were normal. Analysis of cerebrospinal fluid showed a clear and colourless fluid containing $370 \times 10^{6}$ white cells $(80 \%$ lymphocytes), $207 \times 10^{6}$ red cells $1.8 \mathrm{mg} / \mathrm{l}$ protein, and $3.0 \mathrm{mmol} /$ glucose (serum glucose concentration $4.3 \mathrm{mmol} / \mathrm{l})$. Gram staining was negative. Viral organisms were not found in cerebrospinal fluid or throat swabs. Serological tests for Epstein-Barr, mumps, measles, and rubella viruses showed a significan rise in titres of rubella antibodie only (from $30 \mathrm{IU}$ to $240 \mathrm{IU}$ for IgG and from positive to strongly positive for IgM). Radiology of brain and spinal cord, including magnetic resonance imaging, gave norma results. Visual evoked potentials were within normal limits.

Postvaccination

transverse myelitis was diagnosed, and the patient was treated with intravenous steroids for five days. Improvement was only limited and he had remained paralysed below $\mathrm{T} 6$.

To our knowledge, only three cases of possible myelitis after rubella vaccination have been described. ${ }^{1-3}$ Little is known about the pathogenesis of postvaccination transverse myelitis, and we have no information to suggest that the risk increases with concurrent administration of other live viruses, or in those already immune. Antibody status should be checked before immunisation and, if possible, only the required vaccine should be given

1 Fenichel GM. Neurological complications of immunisation. Ann Neurol 1982;12:1 19-28. 2 Holt S, Hudgkins D, Krishnan KR, Critchley EMR. Diffuse myelitis associated with rubella vaccination. $B M F$ f 1976;ii:1037-8.

3 Behan PO. Diffuse myelitis associated with rubella vaccination. $B M \mathcal{F} 1977 ;$ ii: 166 .

\section{Exacerbation of idiopathic Parkinson's disease by naproxen}

Drs S Shaunak, P Brown, and J A Morgan-Hughes (National Hospital for Neurology and Neurosurgery, London WC1N 3BG) write: A 74 year old woman with a 10 year history of idiopathic Parkinson's disease was prescribed naproxen $250 \mathrm{mg}$ three times a day fo musculoskeletal pain. Her symptoms had previously been well controlled with co-beneldopa (levodopa and benserazide hydrochloride) $375 \mathrm{mg}$ a day, selegiline $10 \mathrm{mg}$ a day, and propranolol $60 \mathrm{mg}$ a day. Shortly after starting naproxen treatment she became immobile and more tremulous, subsequently requiring admission to hospital. Examination showed generalised rigidity and bradykinesia and a severe bilateral resting tremor. She was unable to rise from a chair or walk without help. Results of routine blood tests showed no abnormality and a computed tomogram looked normal.

Naproxen treatment was stopped, and her symptoms improved appreciably such that she became mobile and able to care for herself. Six weeks later, and with her informed consent, she was rechallenged with the drug at its original dosage. Within 24 hours she developed considerable tremor, rigidity, and immobility; formal assessment by means of the King's College Parkinson's disease rating scale confirmed a clear deterioration in her Parkinson's disease (the time taken for her to walk 40 metres unaided trebled after the challenge). Recovery occurred within 24 hours of stopping naproxen treatment, and she was discharged taking unchanged doses of co-beneldopa and selegiline.

The drug manufacturers have received no similar reports (Syntex Pharmaceuticals, personal communication), but the Committee on Safety of Medicines has documented one case of parkinsonism associated with a combined naproxenmisoprostol preparation, and 12 reports of tremor or ataxia precipitated by naproxen (personal communication). There are several published reports of extrapyramidal syndromes in patients taking non-steroidal anti-inflammatory drugs, ${ }^{12}$ but few specifically of parkinsonism. Parkinsonian symptoms have been worsened by sulindac in a patient already receiving a levodopa and carbidopa preparation, ${ }^{3}$ and acute parkinsonism associated with flurbiprofen has been described in a previously well patient, in whom positron emission tomograms suggested subclinical nigral cell loss. ${ }^{4}$ Conversely, diflunisal has been reported to improve symptoms in six patients with Parkinson's disease.' These cases suggest that nonsteroidal anti-inflammatory drugs may influence striatal activity, although the mechanism of such an effect remains obscure.

Non-steroidal anti-inflammatory drugs such as naproxen are widely prescribed in general practice, and many can now be bought over the counter. Physicians should be aware that these drugs may exacerbate Parkinson's disease.

1 Wood N, Pall PS, Williams AC, Dieppe C. Extrapyramidal reactions to antiinflammatory drugs. I Neurol Neurosurg Psychiatry 1988;51:731-2.

2 Cremona-Barbaro A. Extrapyramida symptoms following mefenamic acid. $f(R$ Soc Med 1983;76:435.

3 Sandyk R, Gillman MA. Acute exacerbation of Parkinson's disease with sulindac. Ann Neurol 1985;17:104-5.

4 Enevoldson TP, Wiles CM. Acute parkinsonism associated with flurbiprofen. $B M F$ 1990;300:540-1.

5 Anderson CB, Larson EJ. Diflunisal in idiopathic Parkinson's disease. Neurology 1984;34:400. 